\title{
Radiometric Quality of the MODIS Bands at 667 and $678 \mathrm{~nm}$
}

\author{
Gerhard Meister $^{a}$, Bryan A. Franz ${ }^{a}$ \\ ${ }^{a}$ NASA, Ocean Biology Processing Group, Code 614.2, Goddard Space Flight Center, \\ Greenbelt, MD 20771, USA;
}

\begin{abstract}
The MODIS instruments on Terra and Aqua were designed to allow the measurement of chlorophyll fluorescence effects over ocean. The retrieval algorithm is based on the difference between the water-leaving radiances at $667 \mathrm{~nm}$ and $678 \mathrm{~nm}$. The water-leaving radiances at these wavelengths are usually very low relative to the topof-atmosphere radiances. The high radiometric accuracy needed to retrieve the small fluorescence signal lead to a dual gain design for the 667 and $678 \mathrm{~nm}$ bands. This paper discusses the benefits obtained from this design choice and provides justification for the use of only one set of gains for global processing of ocean color products. Noise characteristics of the two bands and their related products are compared to other products of bands from $412 \mathrm{~nm}$ to $2130 \mathrm{~nm}$. The impact of polarization on the two bands is discussed. In addition, the impact of stray light on the two bands is compared to other MODIS bands.
\end{abstract}

Keywords: remote sensing, scanners, on-orbit calibration

\section{INTRODUCTION}

Two units of the Moderate-Resolution Imaging Spectroradiometer (MODIS) ${ }^{1}$ are currently in operation, providing global coverage of top-of-atmosphere (TOA) radiances from $412 \mathrm{~nm}$ to $14200 \mathrm{~nm}$. The first unit was launched in December 1999 on NASA's Earth Observing System (EOS) Terra satellite, the second on the Aqua satellite in May 2002. MODIS has 36 spectral bands on four different focal planes. The Ocean Biology Processing Group (OBPG) at NASA uses bands 8-16 with center wavelengths from $412 \mathrm{~nm}$ to $869 \mathrm{~nm}$ to produce the standard ocean color data products. ${ }^{2}$ The basic ocean color products are water-leaving radiances from bands 8-14, see table 1. Bands 15 and $16(748 \mathrm{~nm}$ and $869 \mathrm{~nm})$ are used in the atmospheric correction process. All ocean color products are derived from the water-leaving radiances. Recently, the OBPG included ocean color products from bands 1 , 3 , and 4. More information on the ocean color products provided by the OBPG can be found at their website: http:oceancolor.gsfc.nasa.gov.

MODIS bands 8-12 and 15-16 each have 10 independent detectors, aligned in the along-track direction. MODIS is a scanning radiometer with a two-sided primary mirror, so every scan line consists of 10 lines in the along-track direction (one for each detector) for a certain mirror side (adjacent scan lines are from alternating mirror sides). The detectors of each band are shielded by a spectral filter that only transmits light in a certain wavelength range (e.g. from $662 \mathrm{~nm}$ to $672 \mathrm{~nm}$ for band 13 ; from $673 \mathrm{~nm}$ to $683 \mathrm{~nm}$ for band 14 ).

MODIS was the first instrument capable of measuring chlorophyll fluorescence from low earth orbits. ${ }^{3}$ Bands 13 and 14 are very close spectrally, but band 14 is within the spectral region influenced by chlorophyll fluorescence. ${ }^{4}$ The fluorescence line height (FLH) product is calculated based on the difference in the water-leaving radiances of these two bands. Noise limitations were an important consideration in the design of these two bands, because the difference of the water-leaving radiances of these two bands is very small compared to the top-of-atmosphere (TOA) radiance (usually much less than 1\%).

Further author information:

G.M.: E-mail: Gerhard.Meister@nasa.gov

Table 1. MODIS ocean color bands and their center wavelengths $\lambda$. Band 6 is not listed here because 8 of its 10 detectors are defunct on MODIS Aqua.

\begin{tabular}{|c|c|c|c|c|c|c|c|c|c|c|c|c|c|c|c|}
\hline Band number & 1 & 2 & 3 & 4 & 5 & 7 & 8 & 9 & 10 & 11 & 12 & 13 & 14 & 15 & 16 \\
\hline$\lambda[\mathrm{nm}]$ & 645 & 869 & 469 & 555 & 1240 & 2135 & 412 & 443 & 488 & 531 & 547 & 667 & 678 & 748 & 869 \\
\hline
\end{tabular}




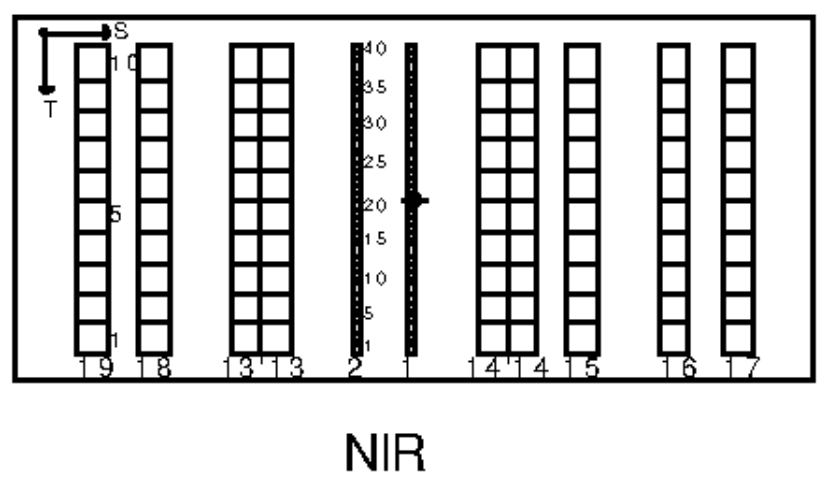

Figure 1. Layout of the MODIS NIR focal plane. Scan direction is to the right (S), track direction towards the bottom (T). Bands 15-19 have one line of 10 detectors each, bands 13 and 14 each have two lines of 10 detectors. Image taken from MODIS Geolocation ATBD. ${ }^{6}$

The design of band 13 (and band 14) is special in that, under the filter, there are two parallel lines of 10 detectors each, see Fig. 1. These two lines of detectors are summed using a time delay and integration (2:1 TDI) approach (the SeaWiFS sensor uses 4:1 TDI, see Eplee et al. ${ }^{5}$ ). For band 13, there are two sets of radiances transmitted from the satellite to the ground. They are called 'Band 13H' and 'Band 13L'. The signal after TDI is split into two signals, and different gains are applied to the two signals (private communication from the MODIS Characterization Support Team (MCST)). The higher gain of band 13H increases the radiometric resolution of that band (smaller radiance increment per $\mathrm{dn}$, see Lsat/4000 in table 2), but reduces the dynamic range (see Lsat in table 2).

This paper evaluates the benefits of this particular design, and describes the reasons why the OBPG uses only the 13L and 14L bands for the ocean color processing. Furthermore, noise, stray light, and polarization characteristics of these two bands are evaluated.

\section{SIGNAL-TO-NOISE RATIO}

The signal-to-noise ratio (SNR) at typical radiances (Ltyp) as measured prelaunch is shown in table 2.

The improvement in SNR is only about $10 \%$ for band $13 \mathrm{H}$ vs $13 \mathrm{~L}$, and $25 \%$ for band $14 \mathrm{H}$ vs $14 \mathrm{~L}$. These are rather modest improvements. At Ltyp, the SNR of band 13L translates into a noise of $0.071 \%$, the SNR of band $13 \mathrm{H}$ into a noise of $0.064 \%$, an improvement of only $0.007 \%$. For band 14 , the improvement is only $0.013 \%$. Most other uncertainty sources (calibration, stray light, polarization, etc.) usually contribute on the order of at least $0.1 \%{ }^{7-9}$ The advantage of the higher SNR of bands $13 \mathrm{H}$ and $14 \mathrm{H}$ is too small to have a meaningful impact.

A similar argument can be made for the decrease in the radiance increment per dn for the high gain bands versus the low gain bands: the radiance increment of band 13L is about $0.09 \%$ of Ltyp, about $0.05 \%$ for band $13 \mathrm{H}$.

Table 2. Radiometric characteristics of MODIS Aqua bands 13 and 14. Saturation radiances Lsat are from granule shown in Fig. 2. Ltyp are from MODIS specifications. SNR were provided by MCST [private communication]. Noise equivalent radiances $(\mathrm{NedL})$ were calculated using NedL $=\mathrm{SNR} /$ Ltyp. Lsat/4000 is an estimate of the radiance increment per dn (MODIS is a 12 bit sensor $\left(2^{12}=4096\right)$, dark current is on the order of $100 \mathrm{dn}$ and must be subtracted, so maximum dn is about 4000). Radiance unit for Lsat, Ltyp, and NedL is $\mathrm{mW} / \mathrm{cm}^{2} / \mu \mathrm{m} / \mathrm{sr}$.

\begin{tabular}{|l|c|c|c|c|}
\hline MODIS band & $13 \mathrm{~L}$ & $13 \mathrm{H}$ & $14 \mathrm{~L}$ & $14 \mathrm{H}$ \\
\hline Lsat & 3.58 & 1.70 & 3.56 & 1.28 \\
\hline Ltyp & 0.95 & 0.95 & 0.87 & 0.87 \\
\hline SNR at Ltyp & 1405 & 1551 & 1507 & 1885 \\
\hline NedL & 0.00068 & 0.00061 & 0.00058 & 0.00046 \\
\hline Lsat/4000 & 0.00090 & 0.00043 & 0.00089 & 0.00032 \\
\hline
\end{tabular}



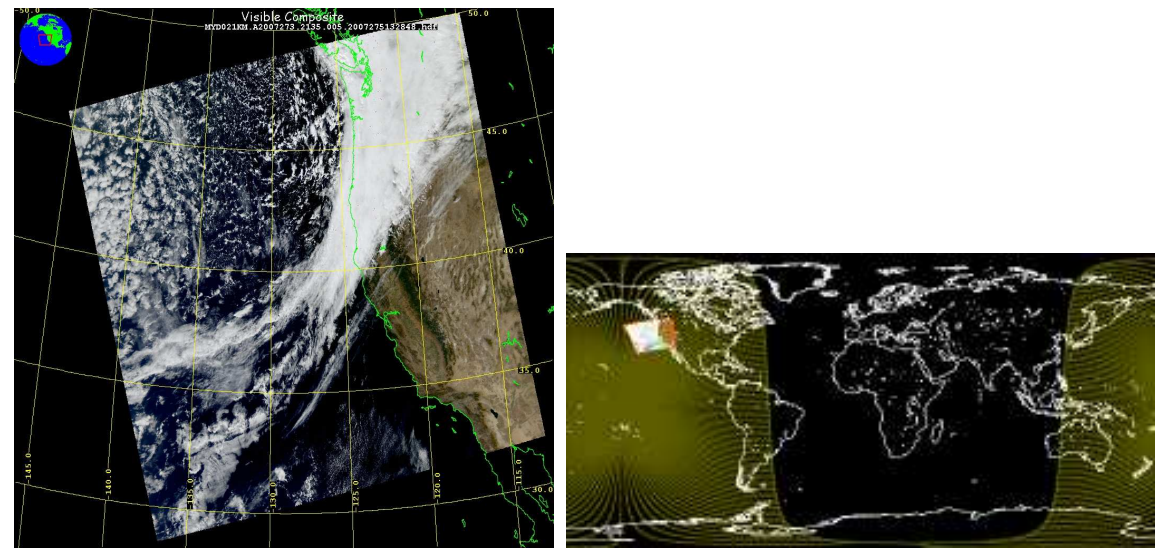

Figure 2. Left: True color image of the test granule used in section 3. Image provided by LAADS web. Right: Location of the granule on the globe. Image provided by OBPG.
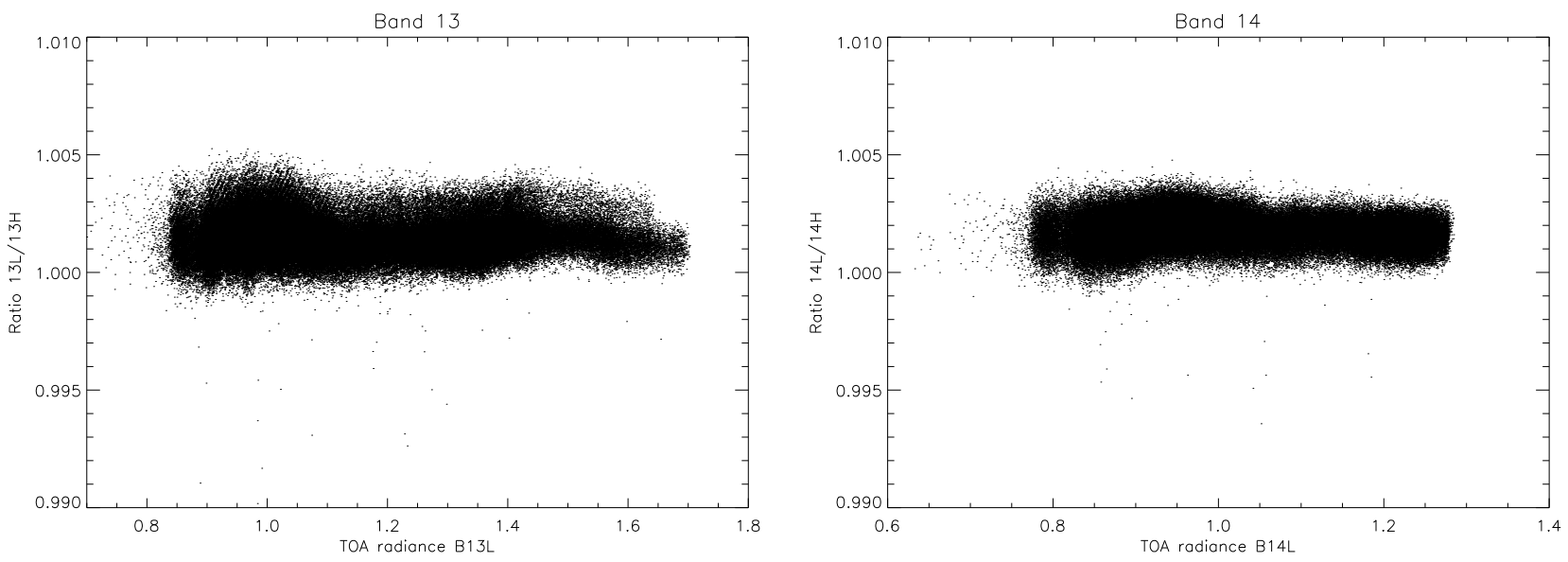

Figure 3. Left: ratio of the low gain to the high gain L1B radiances for band 13. Right: same for band 14 .

This corresponds to an improvement of $0.04 \%$. For band 14, the improvement is $0.07 \%$. These improvements are small compared to most other uncertainty sources.

The radiance increments per dn decrease by more than $50 \%$ for the high gain bands relative to the low gain bands. These differences are much larger than the change in SNR, which implies that noise of the low gain bands is not dominated by quantization noise.

\section{ANALYSIS OF IMAGE DATA}

MODIS Aqua granule A20072732135 (see Fig.2) was used to investigate the benefits of substituting bands 13H and $14 \mathrm{H}$ for bands $13 \mathrm{~L}$ and $14 \mathrm{~L}$.

From this granule, only pixels were selected where the flags ATMFAIL, LAND, STRAYLIGHT, CLDICE, CHLFAIL, MODGLINT were not set, and also where band 14H did not saturate (a saturation threshold of 1.25 $\mathrm{mW} / \mathrm{cm}^{2} / \mu \mathrm{m} / \mathrm{sr}$ was chosen for band $14 \mathrm{H}, 14 \mathrm{H}$ always saturated when $13 \mathrm{H}$ saturated). The ratio of the low gain to the high gain L1B radiances for the selected pixels are shown in Fig.3 as a function of low gain radiance, for each band 13 and 14.

It can be seen that there is some variation in the ratio of about $\pm 0.2 \%$, and a bias of about $0.2 \%$, with low-gain bands higher than the high-gain bands. 

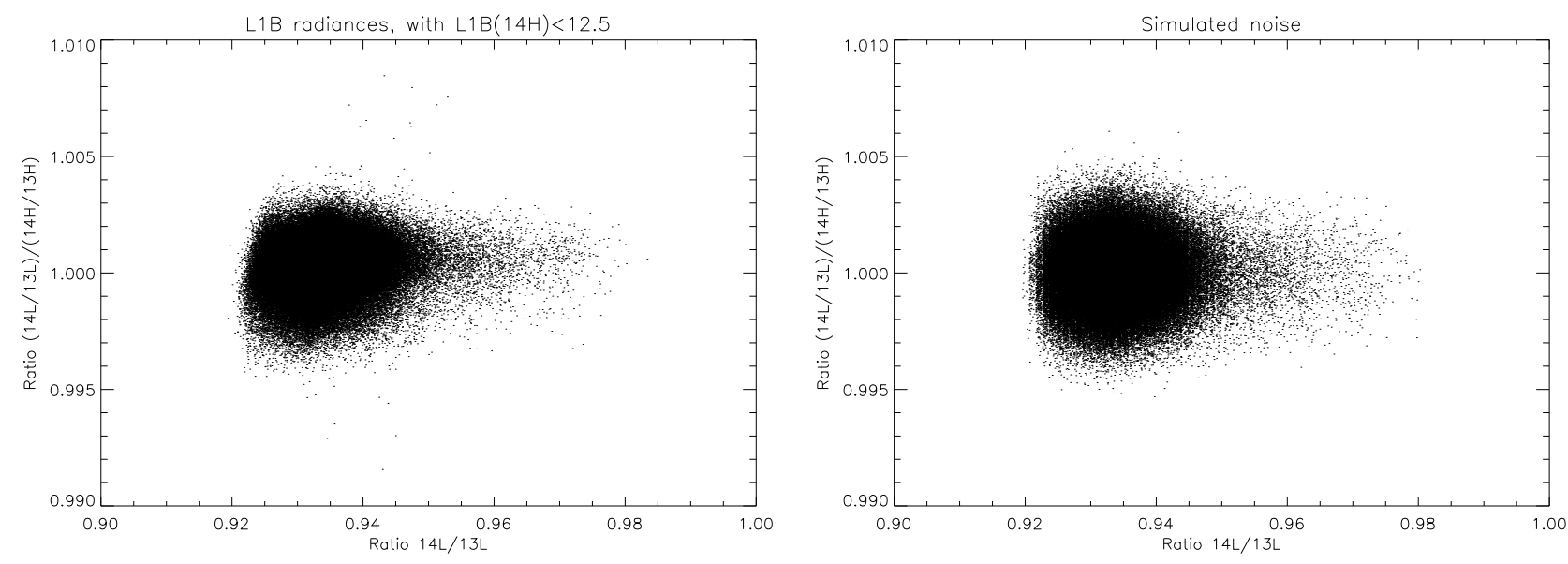

Figure 4. Left: Measured ratio of $14 \mathrm{~L} / 13 \mathrm{~L}$ over $14 \mathrm{H} / 13 \mathrm{H}$ as a function of $14 \mathrm{~L} / 13 \mathrm{~L}$. Right: Simulated ratio of $14 \mathrm{~L} / 13 \mathrm{~L}$ over $14 \mathrm{H} / 13 \mathrm{H}$ as a function of $14 \mathrm{~L} / 13 \mathrm{~L}$. Distribution of values along x-axis $(14 \mathrm{~L} / 13 \mathrm{~L})$ is taken from the plot on the right, noise was added to ratio of $14 \mathrm{~L} / 13 \mathrm{~L}$ over $14 \mathrm{H} / 13 \mathrm{H}$ (setting $14 \mathrm{H} / 13 \mathrm{H}$ equal to $14 \mathrm{~L} / 13 \mathrm{~L}$ before noise was added), using SNR from table 2.

Presumably, if the high gain bands were to be used instead of the low gain bands, they would be used in both bands 13 and 14. The FLH algorithm depends on the difference of the radiance between the two bands. Fig.4 shows the ratio of $14 \mathrm{~L} / 13 \mathrm{~L}$ over $14 \mathrm{H} / 13 \mathrm{H}$ as a function of $14 \mathrm{~L} / 13 \mathrm{~L}$.

It can be seen that there is almost no bias in the measured ratios (the mean of $14 \mathrm{~L} / 13 \mathrm{~L}$ over $14 \mathrm{H} / 13 \mathrm{H}$ is 1.0002), but there is a noticeable variation (standard deviation is 0.0011 ). In order to test whether this variation can be explained by the SNR obtained with the prelaunch SNR, we simulated this ratio, assuming the SNR at Ltyp for all radiance levels (this assumption underestimates the noise, because most radiances where $14 \mathrm{H}$ does not saturate are below Ltyp). The results are shown in Fig. 4 as well.

The simulated ratios are very similar to the measured ratios. The simulated ratios have a slightly larger spread (standard deviation of all points is 0.0013). One possible explanation is that the noise in the measured high gain band radiances is correlated with the noise in the low gain band radiances (there is no correlation in the simulated radiances). Considering that for each band, both the high gain and the low gain radiances are extracted from the same data source (the sum of the two TDI detectors of each band), a certain amount of correlation in the ratios $14 \mathrm{~L} / 13 \mathrm{~L}$ over $14 \mathrm{H} / 13 \mathrm{H}$ should be expected.

Fig.5 shows the FLH values from a subscene of the granule of Fig.2 (from the center of the granule, the US west coast is part of the subscene) processed using SeaDAS 5.1.3 with the high gain bands (left) versus the low gain bands (right). SeaDAS usually calculates the $\mathrm{nLw}$ of bands 13 and 14 using the low gain bands. To create the $n L w$ using the high gain bands for these plots, the low gain bands in the L1B file were overwritten with the high gain bands, after adjusting the mean L1B radiance of the high gain bands to the mean L1B radiance of the low gain bands.

The images look very similar, with no apparent difference in the noise characteristics between the high gain and low gain image. The high gain image has stronger striping. For the low gain bands, the OBPG uses a correction based on TOA residuals. ${ }^{10}$ This correction has not been calculated for the high gain bands, instead the low gain correction was applied. It is possible that the striping would be similar in both images if corrections derived for the high gain bands were applied, although the corrections for high and low gain are expected to be very similar.

Note that the low-gain bands provide more spatial coverage than the high-gain bands (black areas are larger in the plot on the left in Fig.5 than in the right plot, especially in the coastal area with high FLH values (red); San Francisco Bay (at $37^{\circ}$ latitude and $122^{\circ}$ longitude) is partially retrieved with the low-gain bands, but not at all with the high gain bands, due to the lower saturation limits). Coverage in coastal areas is an important 

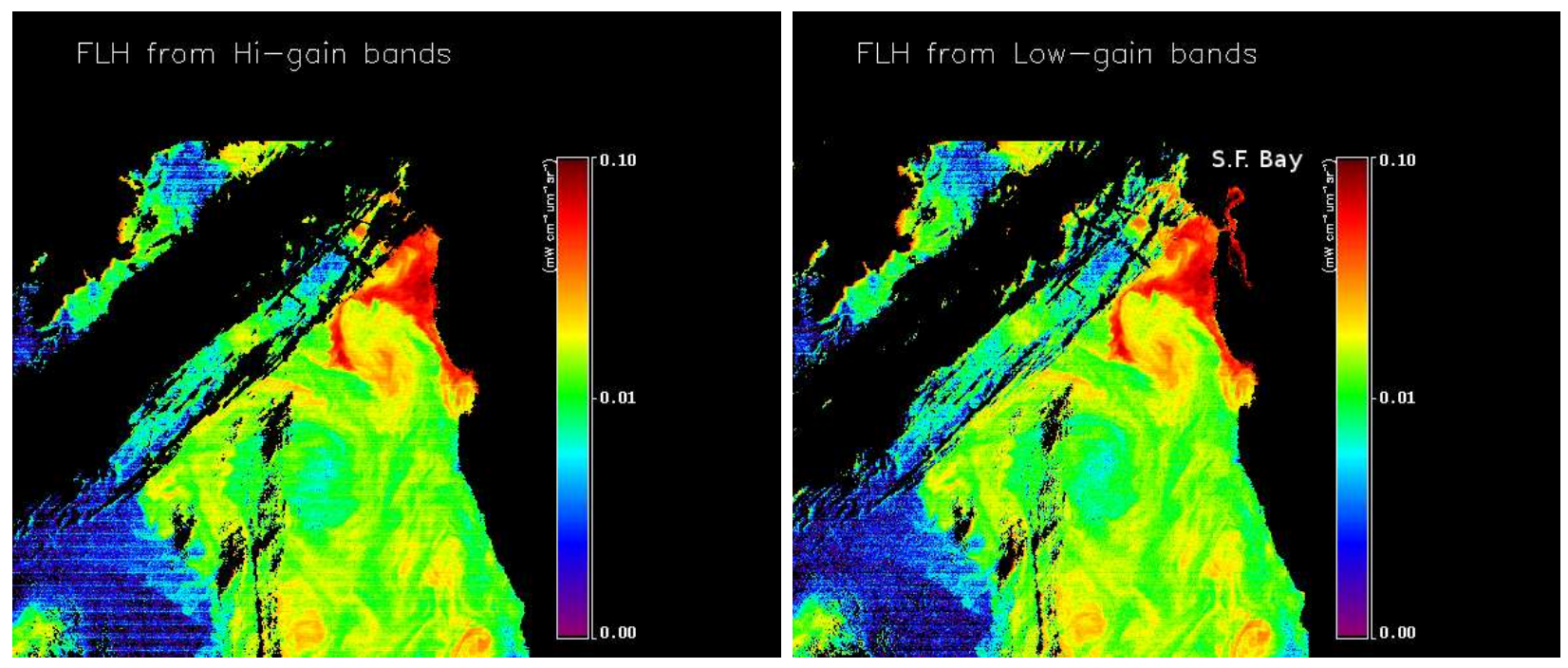

Figure 5. FLH product for a subscene of granule shown in Fig. 2. Left: using 13H and 14H. Right: using 13L and 14L.

aspect of an ocean color sensor, and lead the OBPG to choose the low-gain bands for the NASA ocean color processing instead of the high-gain bands. Table 2 shows that the dynamic range advantage of the low gain bands over the high gain bands quite large, especially for band 14. For band 14H, Lsat is very close to the mean value of the scene analyzed in table 4 below.

\section{STATISTICAL NOISE ANALYSIS}

The MODIS instrument noise was originally determined using solar diffuser measurements. ${ }^{11}$ The results are expected to give a good approximation to the true instrument noise because the solar diffuser is a homogeneous surface that contributes only marginally to the measured noise. In this section, the noise of earth-viewing scenes is analyzed. The resulting noise is necessarily higher than the instrument noise, because earth scenes generally contain significant variation. The analysis in this section was performed with data from reprocessing R2010.0 for both MODIS instruments and SeaWiFS.

The idea behind our approach is that open ocean scenes are often homogeneous on a $5 \mathrm{~km}$ scale. The main radiance variation over open oceans is introduced by clouds. The standard OBPG L3 masks will remove cloud pixels (CLDICE mask) and pixels adjacent to clouds (STRAYLIGHT mask). By analyzing the variation in 5x5 pixel boxes where no masks are set, a large part of the true variability of the earth scene is removed.

The algorithm consists of the following steps:

- select pixels from common region in L2 file, mask with L3 masks

- find number of pixels (N) that are the center of a completely unmasked $5 \times 5$ box, use index $i$ with $1 \leq i \leq N$ to index them

- for each pixel $i$ (at row $x$, line $y$, with product value $p_{i}=p(x, y)$ ), calculate

$$
\begin{aligned}
d_{i}^{m} & =\frac{1}{25} \cdot \sum_{j=x-2, k=y-2}^{j=x+2, k=y+2} p(j, k) \\
d_{i} & =p_{i}-d_{i}^{m}
\end{aligned}
$$

To estimate the noise due to instrument noise, a simulated data set was created, using the same $5 \times 5$ boxes as in the algorithm above, but calculating $d_{i}$ with

$$
d_{i}=p_{i}^{s}-d_{i}^{m}
$$



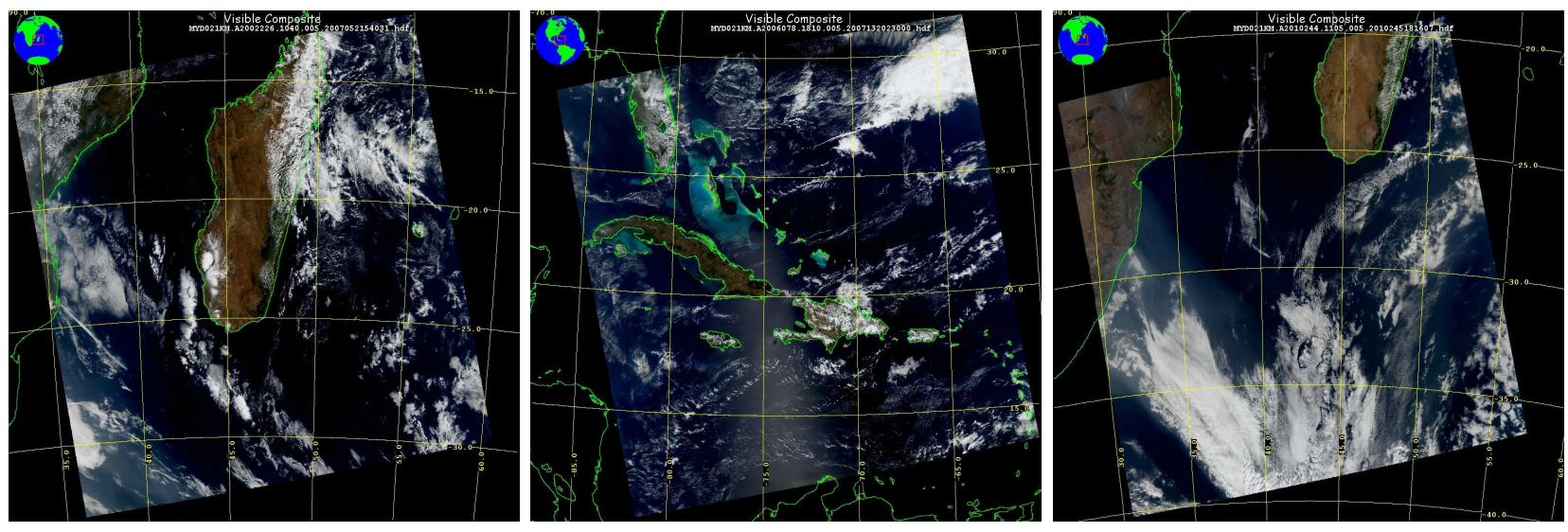

Figure 6. Left: True color images of the MODIS Aqua granules used in section 4. Images provided by LAADS web.

where $p_{i}^{s}$ was calculated by adding noise to $d_{i}^{m}$, using SNR provided Xiong et al., $2010^{11}$ (this was done only for the TOA radiances of the ocean bands, the SNR provided by Xiong et al. for the land bands are not applicable to typical TOA ocean radiances).

Granules were chosen for the years 2002, 2006, and 2010. The MODIS Aqua granules are shown in Fig. 6, they all contain a relatively high amount of cloud free open ocean data. For each year, overlapping MODIS Terra granules were chosen less than 30 hours apart. Only pixels from a common area were evaluated. The number $N$ of non-masked $5 \times 5$ boxes in each granule varies from 22,000 to 170,000 for the MODIS granules (17,000 for the SeaWiFS scene). The median of $\left|d_{i} / d_{i}^{m}\right|$ provides an estimate of the typical relative variation for each product. The standard deviation of $d_{i}$ and the mean product value are given as well. The results are shown in tables 3 to 5 .

For a cloud free open ocean scene with natural variation, the expected difference of the TOA radiance (Lt) at $412 \mathrm{~nm}$ of a pixel to the mean of the $5 \times 5$ box it is centered on ranges from $0.11 \%$ to $0.14 \%$ for MODIS on both Aqua and Terra, and SeaWiFS. This is about twice of the result expected from the instrument noise itself (compare with 0.06\%-0.07\% from column Aqua-sim. in table 3 and 5, which was calculated with equation 3). At $865 \mathrm{~nm}$, the expected difference is typically about $0.5 \%$, which is ten times higher than the result expected from pure instrument noise alone (compare with $0.05 \%$ from column Aqua-sim.). Absolute differences decrease with wavelength (see columns with standard deviation of $d_{i}$ ), but relative differences (see columns with $\left|d_{i} / d_{i}^{m}\right|$ ) increase, due to the significant drop in radiance with wavelength. The latter applies to the measured data only, the relative differences of the simulated data (using instrument noise only) do no change significantly with wavelength.

To compare the noise characteristics of the high gain to the low gain bands, the results for bands 13 and 14 were also calculated for those $5 \mathrm{x} 5$ boxes were band $14 \mathrm{H}$ did not saturate. These results are provided as Lt_667L, Lt_667H, Lt_678L and Lt_678H in tables 3 to 5 . They are very similar to the Lt_667 and Lt_678 results (which are calculated with the low gain bands, but include a larger number of pixels because band $14 \mathrm{H}$ saturation is not a criterion), except for Aqua in table 4, where band $14 \mathrm{H}$ saturation eliminated about half of the $5 \mathrm{x} 5$ boxes. The lower sensor noise of the high gain bands does not show a measurable improvement with the metric used here for the TOA radiances of bands 13 and 14.

For the $\mathrm{nLw}$ products, MODIS land bands have higher variation than the nearest ocean bands. MODIS Terra $\mathrm{nLw}$ products of both ocean and land bands typically have more noise than those of MODIS Aqua (MODIS Aqua $\mathrm{nLw}$ at $645 \mathrm{~nm}$ is a consistent exception).

SeaWiFS has a higher variation in epsilon (twice of MODIS), which causes the increase in variation of nLw and chlorophyll, see table 4. The Lt variation of SeaWiFS in the NIR is not significantly higher, which is unexpected. Maybe natural variability is similar to sensor noise. 
The noise in the MODIS SST4 products is significantly lower than in the MODIS SST products, for both MODIS Aqua and Terra. Note that only ocean color flags were used to analyze these two products, no sea surface temperature specific flags or masks.

\section{STRAY LIGHT ASPECTS}

The same methodology as in Meister et al., $2010^{8}$ was used to compare the stray light contamination of bands 13 and 14 to stray light in other MODIS bands. The same granules as in tables 3 to 5 were used. It can be seen in Fig. 7 that there is no obvious difference in stray light response of bands 13 and 14 to band 1 (which is at a

Table 3. Mean product value, standard deviation of $d_{i}$, and median of $\left|d_{i} / d_{i}^{m}\right|$ for MODIS Terra and Aqua for 2002 granules T20022270705 and A20022261040. A common region with latitudes from $-30.10^{\circ}$ to $-25.50^{\circ}$ and longitudes from $48.50^{\circ}$ to $56.00^{\circ}$ was used in each granule. Radiance and FLH units are $\mathrm{mW} / \mathrm{cm}^{2} / \mu \mathrm{m} / \mathrm{sr}$, chlorophyll units are $\mathrm{mg} / \mathrm{m}^{3}$, aerosol optical thickness (AOT) and epsilon are dimensionless, sea surface temperature (SST) units are degrees Celsius. Products with the suffix 'L' or 'H' were evaluated for regions were band $14 \mathrm{H}$ did not saturate.

\begin{tabular}{|c|c|c|c|c|c|c|c|}
\hline \multirow[t]{2}{*}{ Product } & \multicolumn{2}{|c|}{ Mean of $p_{i}$} & \multicolumn{2}{|c|}{ Stdev. of $d_{i}$} & \multicolumn{3}{|c|}{ Median of $\left|d_{i} / d_{i}^{m}\right| \cdot 100$} \\
\hline & Terra & Aqua & Terra & Aqua & Terra & Aqua & Aqua-sim. \\
\hline Lt_412 & 6.006 & 6.470 & 0.017 & 0.015 & 0.11 & 0.13 & 0.06 \\
\hline Lt_443 & 5.235 & 5.725 & 0.019 & 0.015 & 0.13 & 0.14 & 0.04 \\
\hline Lt_469 & 4.834 & 5.301 & 0.021 & 0.016 & 0.16 & 0.15 & \\
\hline Lt_488 & 3.904 & 4.238 & 0.019 & 0.013 & 0.12 & 0.13 & 0.04 \\
\hline Lt_531 & 2.443 & 2.672 & 0.017 & 0.012 & 0.14 & 0.15 & 0.04 \\
\hline Lt_547 & 2.137 & 2.333 & 0.017 & 0.011 & 0.16 & 0.14 & 0.04 \\
\hline Lt_555 & 2.002 & 2.180 & 0.018 & 0.012 & 0.24 & 0.21 & \\
\hline Lt_645 & 0.975 & 1.042 & 0.016 & 0.011 & 0.40 & 0.29 & \\
\hline Lt_667 & 0.848 & 0.911 & 0.015 & 0.010 & 0.34 & 0.22 & 0.05 \\
\hline Lt_667L & 0.848 & 0.909 & 0.015 & 0.009 & 0.38 & 0.21 & \\
\hline Lt_667H & 0.848 & 0.907 & 0.015 & 0.009 & 0.38 & 0.21 & \\
\hline Lt_678 & 0.792 & 0.843 & 0.015 & 0.010 & 0.34 & 0.22 & 0.04 \\
\hline Lt_678L & 0.792 & 0.842 & 0.015 & 0.009 & 0.37 & 0.21 & \\
\hline Lt_678H & 0.791 & 0.840 & 0.015 & 0.009 & 0.37 & 0.21 & \\
\hline Lt_748 & 0.517 & 0.535 & 0.013 & 0.009 & 0.48 & 0.29 & 0.04 \\
\hline Lt_859 & 0.274 & 0.268 & 0.010 & 0.008 & 0.86 & 0.51 & \\
\hline Lt_869 & 0.265 & 0.256 & 0.010 & 0.008 & 0.78 & 0.43 & 0.05 \\
\hline Lt_ 1240 & 0.075 & 0.053 & 0.005 & 0.004 & 3.04 & 2.42 & \\
\hline Lt_2130 & 0.009 & 0.006 & 0.001 & 0.001 & 5.60 & 5.04 & \\
\hline nLw_412 & 1.613 & 1.546 & 0.028 & 0.028 & 1.04 & 1.01 & \\
\hline nLw_443 & 1.557 & 1.500 & 0.024 & 0.025 & 0.88 & 0.90 & \\
\hline nLw_469 & 1.508 & 1.499 & 0.024 & 0.023 & 1.00 & 0.88 & \\
\hline nLw_488 & 1.154 & 1.161 & 0.014 & 0.014 & 0.75 & 0.72 & \\
\hline nLw_531 & 0.419 & 0.414 & 0.008 & 0.009 & 1.32 & 1.32 & \\
\hline nLw_547 & 0.314 & 0.313 & 0.008 & 0.008 & 1.69 & 1.59 & \\
\hline nLw_555 & 0.286 & 0.262 & 0.011 & 0.011 & 2.53 & 2.64 & \\
\hline nLw_645 & 0.045 & 0.031 & 0.007 & 0.006 & 9.60 & 13.66 & \\
\hline nLw_667 & 0.023 & 0.025 & 0.003 & 0.003 & 8.41 & 7.12 & \\
\hline nLw_678 & 0.027 & 0.025 & 0.003 & 0.002 & 6.17 & 6.27 & \\
\hline chlor_a & 0.086 & 0.092 & 0.004 & 0.004 & 2.95 & 2.62 & \\
\hline aot_869 & 0.064 & 0.053 & 0.006 & 0.005 & 2.18 & 2.16 & \\
\hline epsilon & 1.027 & 1.025 & 0.008 & 0.008 & 0.43 & 0.43 & \\
\hline $\mathrm{flh}$ & 0.004 & 0.002 & 0.002 & 0.001 & 15.12 & 23.78 & \\
\hline sst & 21.423 & 20.784 & 0.193 & 0.121 & 0.59 & 0.39 & \\
\hline sst4 & 21.846 & 21.161 & 0.087 & 0.093 & 0.24 & 0.27 & \\
\hline
\end{tabular}


similar wavelength), but that generally stray light effects increase dramatically with wavelength (relative to the typical ocean radiances). This may be one aspect that increases the noise with wavelength shown in tables 3 to 5.

The stars in Fig. 7 qualitatively relate the stray light contamination to the mean radiance. It can be seen that stray light effects in pixels used for ocean color processing (data to the right of the vertical dashed line) is contaminated by stray light on the order of about $1 \%$ for $412 \mathrm{~nm}$, up to about $5 \%$ for the red bands, and on the order of $10 \%$ or more for $1240 \mathrm{~nm}$ and $2130 \mathrm{~nm}$. Note that the atmospheric correction algorithm used in the NASA ocean color processing removes a large amount of residual stray light contamination by assuming it is

Table 4. Mean product value, standard deviation of $d_{i}$, and median of $\left|d_{i} / d_{i}^{m}\right|$ for MODIS Terra and Aqua for 2006 granules T20060781505 and A20060781810. Equivalent data are given for SeaWiFS for 2006 MLAC scene S2006078173013 as well, but note that SeaWiFS center wavelengths $(412 \mathrm{~nm}, 445 \mathrm{~nm}, 490 \mathrm{~nm}, 510 \mathrm{~nm}, 555 \mathrm{~nm}, 670 \mathrm{~nm}, 765 \mathrm{~nm}, 865 \mathrm{~nm})$ are not identical to MODIS center wavelengths (e.g., data for SeaWiFS Lt_510 is provided under Lt_531). A common region with latitudes from $22.06^{\circ}$ to $24.12^{\circ}$ and longitudes from $-67.24^{\circ}$ to $70.50^{\circ}$ was used in each granule. See table 3 for units.

\begin{tabular}{|c|c|c|c|c|c|c|c|c|c|}
\hline \multirow[t]{2}{*}{ Product } & \multicolumn{3}{|c|}{ Mean of $p_{i}$ for } & \multicolumn{3}{|c|}{ Stdev. of $d_{i}$} & \multicolumn{3}{|c|}{ Median of $\left|d_{i} / d_{i}^{m}\right| \cdot 100$} \\
\hline & Terra & Aqua & SeaW. & Terra & Aqua & SeaW. & Terra & Aqua & SeaW \\
\hline Lt_412 & 8.864 & 9.631 & 9.986 & 0.026 & 0.022 & 0.019 & 0.14 & 0.11 & 0.11 \\
\hline Lt_443 & 7.623 & 8.475 & 8.657 & 0.026 & 0.024 & 0.020 & 0.12 & 0.12 & 0.11 \\
\hline Lt_469 & 6.914 & 7.700 & & 0.031 & 0.026 & & 0.14 & 0.12 & \\
\hline Lt_488 & 5.480 & 6.123 & 6.056 & 0.029 & 0.025 & 0.019 & 0.11 & 0.11 & 0.10 \\
\hline Lt_531 & 3.334 & 3.815 & 4.754 & 0.030 & 0.025 & 0.019 & 0.11 & 0.13 & 0.11 \\
\hline Lt_547 & 2.901 & 3.340 & & 0.030 & 0.025 & & 0.13 & 0.14 & \\
\hline Lt_555 & 2.697 & 3.118 & 3.149 & 0.030 & 0.026 & 0.019 & 0.20 & 0.17 & 0.14 \\
\hline Lt_645 & 1.272 & 1.522 & & 0.029 & 0.025 & & 0.27 & 0.32 & \\
\hline Lt_667 & 1.098 & 1.336 & 1.327 & 0.028 & 0.024 & 0.018 & 0.23 & 0.23 & 0.27 \\
\hline Lt_667L & 1.097 & 1.280 & & 0.028 & 0.007 & & 0.27 & 0.19 & \\
\hline Lt_667H & 1.098 & 1.279 & & 0.028 & 0.007 & & 0.27 & 0.19 & \\
\hline Lt_678 & 1.015 & 1.238 & & 0.028 & 0.024 & & 0.24 & 0.24 & \\
\hline Lt_678L & 1.015 & 1.184 & & 0.028 & 0.006 & & 0.28 & 0.19 & \\
\hline Lt_678H & 1.015 & 1.182 & & 0.028 & 0.006 & & 0.28 & 0.19 & \\
\hline Lt_748 & 0.644 & 0.798 & 0.633 & 0.025 & 0.022 & 0.015 & 0.33 & 0.32 & 0.38 \\
\hline Lt_859 & 0.321 & 0.407 & & 0.021 & 0.018 & & 0.72 & 0.51 & \\
\hline Lt_869 & 0.306 & 0.387 & 0.394 & 0.020 & 0.017 & 0.013 & 0.53 & 0.48 & 0.54 \\
\hline Lt_1240 & 0.060 & 0.079 & & 0.011 & 0.010 & & 3.96 & 1.94 & \\
\hline Lt_2130 & 0.004 & 0.006 & & 0.002 & 0.002 & & 12.38 & 4.90 & \\
\hline nLw_412 & 2.297 & 2.255 & 2.290 & 0.029 & 0.025 & 0.049 & 0.78 & 0.66 & 1.24 \\
\hline nLw_443 & 2.070 & 2.049 & 2.066 & 0.022 & 0.020 & 0.047 & 0.62 & 0.59 & 1.22 \\
\hline nLw_469 & 1.889 & 1.830 & & 0.020 & 0.017 & & 0.69 & 0.57 & \\
\hline nLw_488 & 1.336 & 1.325 & 1.273 & 0.013 & 0.011 & 0.039 & 0.58 & 0.51 & 1.64 \\
\hline nLw_531 & 0.426 & 0.406 & 0.650 & 0.010 & 0.009 & 0.037 & 1.25 & 1.14 & 3.06 \\
\hline nLw_547 & 0.318 & 0.302 & & 0.009 & 0.009 & & 1.57 & 1.45 & \\
\hline nLw_555 & 0.260 & 0.241 & 0.256 & 0.010 & 0.010 & 0.024 & 2.40 & 2.36 & 5.54 \\
\hline nLw_645 & 0.031 & 0.024 & & 0.005 & 0.008 & & 10.45 & 21.06 & \\
\hline nLw_667 & 0.018 & 0.019 & 0.020 & 0.003 & 0.003 & 0.009 & 10.47 & 8.77 & 24.18 \\
\hline nLw_678 & 0.017 & 0.016 & & 0.003 & 0.002 & & 9.53 & 9.15 & \\
\hline chlor_a & 0.048 & 0.043 & 0.039 & 0.003 & 0.003 & 0.008 & 3.31 & 3.24 & 12.27 \\
\hline aot_$\_869$ & 0.029 & 0.042 & 0.036 & 0.005 & 0.004 & 0.003 & 1.29 & 1.00 & 1.47 \\
\hline epsilon & 1.117 & 1.134 & 1.124 & 0.011 & 0.008 & 0.017 & 0.49 & 0.23 & 0.79 \\
\hline flh & 0.002 & 0.001 & & 0.007 & 0.003 & & 38.98 & 171.90 & \\
\hline sst & 25.725 & 25.693 & & 0.163 & 0.139 & & 0.42 & 0.37 & \\
\hline sst4 & 25.723 & 25.868 & & 0.075 & 0.097 & & 0.17 & 0.22 & \\
\hline
\end{tabular}


aerosol radiance. This is evident from Fig. 7: although the Lt products continuously decrease with increasing distance from a cloud, the water-leaving radiances do not show a consistent decrease.

\section{POLARIZATION ASPECTS}

The impact of sensor polarization sensitivity on ocean color products depends on the degree of polarization of the TOA radiances. The average degree of linear polarization $d_{p}$ (as defined in Meister et al., ${ }^{9}$ eq. 14) of the TOA radiances for the red bands is only a little lower than for the green and blue bands, as can be seen in Fig. 8.

Fig. 9 shows the MODIS polarization sensitivity parameters $m_{12}$ and $m_{13}$ derived with the crosscalibration method $^{12}$ and prelaunch ${ }^{9}$ for the end of scan. The end of scan was chosen because the degree of polarization

Table 5. Mean product value, standard deviation of $d_{i}$, and median of $\left|d_{i} / d_{i}^{m}\right|$ for MODIS Terra and Aqua for 2010 granules T20102430740 and A20102441105. A common region with latitudes from $-21.08^{\circ}$ to $-22.40^{\circ}$ and longitudes from $37.72^{\circ}$ to $42.10^{\circ}$ was used in each granule. See table 3 for units.

\begin{tabular}{|c|c|c|c|c|c|c|c|}
\hline \multirow[t]{2}{*}{ Product } & \multicolumn{2}{|c|}{ Mean of $p_{i}$} & \multicolumn{2}{|c|}{ Stdev. of $d_{i}$} & \multicolumn{3}{|c|}{ Median of $\left|d_{i} / d_{i}^{m}\right| \cdot 100$} \\
\hline & Terra & Aqua & Terra & Aqua & Terra & Aqua & Aqua-sim. \\
\hline Lt_412 & 6.575 & 6.622 & 0.016 & 0.015 & 0.14 & 0.13 & 0.07 \\
\hline Lt_ 443 & 5.805 & 5.777 & 0.017 & 0.016 & 0.14 & 0.14 & 0.05 \\
\hline Lt_469 & 5.493 & 5.273 & 0.028 & 0.017 & 0.27 & 0.15 & \\
\hline Lt_488 & 4.479 & 4.272 & 0.017 & 0.014 & 0.16 & 0.12 & 0.05 \\
\hline Lt_531 & 3.009 & 2.778 & 0.017 & 0.013 & 0.24 & 0.13 & 0.04 \\
\hline Lt_547 & 2.690 & 2.440 & 0.018 & 0.014 & 0.27 & 0.15 & 0.04 \\
\hline Lt_555 & 2.523 & 2.274 & 0.019 & 0.014 & 0.37 & 0.22 & \\
\hline Lt_645 & 1.401 & 1.136 & 0.017 & 0.014 & 0.55 & 0.38 & \\
\hline Lt_667 & 1.260 & 0.998 & 0.017 & 0.013 & 0.55 & 0.28 & 0.05 \\
\hline Lt_667L & 1.260 & 0.997 & 0.017 & 0.010 & 0.59 & 0.28 & \\
\hline Lt_667H & 1.260 & 0.996 & 0.018 & 0.010 & 0.59 & 0.28 & \\
\hline Lt_678 & 1.190 & 0.930 & 0.016 & 0.013 & 0.57 & 0.30 & 0.04 \\
\hline Lt_678L & 1.190 & 0.929 & 0.017 & 0.010 & 0.60 & 0.29 & \\
\hline Lt_ $678 \mathrm{H}$ & 1.191 & 0.926 & 0.017 & 0.010 & 0.60 & 0.29 & \\
\hline Lt_748 & 0.860 & 0.613 & 0.015 & 0.012 & 0.73 & 0.40 & 0.04 \\
\hline Lt_859 & 0.533 & 0.335 & 0.012 & 0.010 & 1.01 & 0.65 & \\
\hline Lt_869 & 0.524 & 0.318 & 0.012 & 0.009 & 0.97 & 0.60 & 0.05 \\
\hline Lt_1240 & 0.188 & 0.083 & 0.007 & 0.005 & 1.89 & 1.99 & \\
\hline Lt_2130 & 0.026 & 0.010 & 0.001 & 0.001 & 2.88 & 3.13 & \\
\hline nLw_412 & 1.257 & 1.475 & 0.028 & 0.026 & 1.45 & 1.13 & \\
\hline nLw_443 & 1.253 & 1.320 & 0.025 & 0.022 & 1.19 & 1.02 & \\
\hline nLw_469 & 1.333 & 1.196 & 0.040 & 0.020 & 1.84 & 1.05 & \\
\hline nLw_488 & 0.989 & 0.993 & 0.013 & 0.013 & 0.86 & 0.78 & \\
\hline nLw_531 & 0.422 & 0.425 & 0.008 & 0.008 & 1.26 & 1.10 & \\
\hline nLw_547 & 0.323 & 0.330 & 0.008 & 0.008 & 1.58 & 1.33 & \\
\hline nLw_555 & 0.261 & 0.261 & 0.015 & 0.010 & 4.02 & 2.43 & \\
\hline nLw_645 & 0.041 & 0.041 & 0.005 & 0.006 & 8.89 & 10.15 & \\
\hline nLw_667 & 0.024 & 0.023 & 0.003 & 0.002 & 7.87 & 6.66 & \\
\hline nLw_678 & 0.022 & 0.022 & 0.003 & 0.002 & 7.66 & 6.43 & \\
\hline chlor_a & 0.130 & 0.123 & 0.005 & 0.005 & 2.55 & 2.16 & \\
\hline aot_869 & 0.160 & 0.080 & 0.007 & 0.005 & 1.66 & 1.62 & \\
\hline epsilon & 1.024 & 1.078 & 0.004 & 0.005 & 0.22 & 0.26 & \\
\hline $\mathrm{flh}$ & 0.001 & 0.001 & 0.001 & 0.001 & 52.98 & 43.12 & \\
\hline sst & 24.992 & 25.039 & 0.199 & 0.154 & 0.54 & 0.41 & \\
\hline sst4 & 26.360 & 25.750 & 0.075 & 0.069 & 0.18 & 0.16 & \\
\hline
\end{tabular}



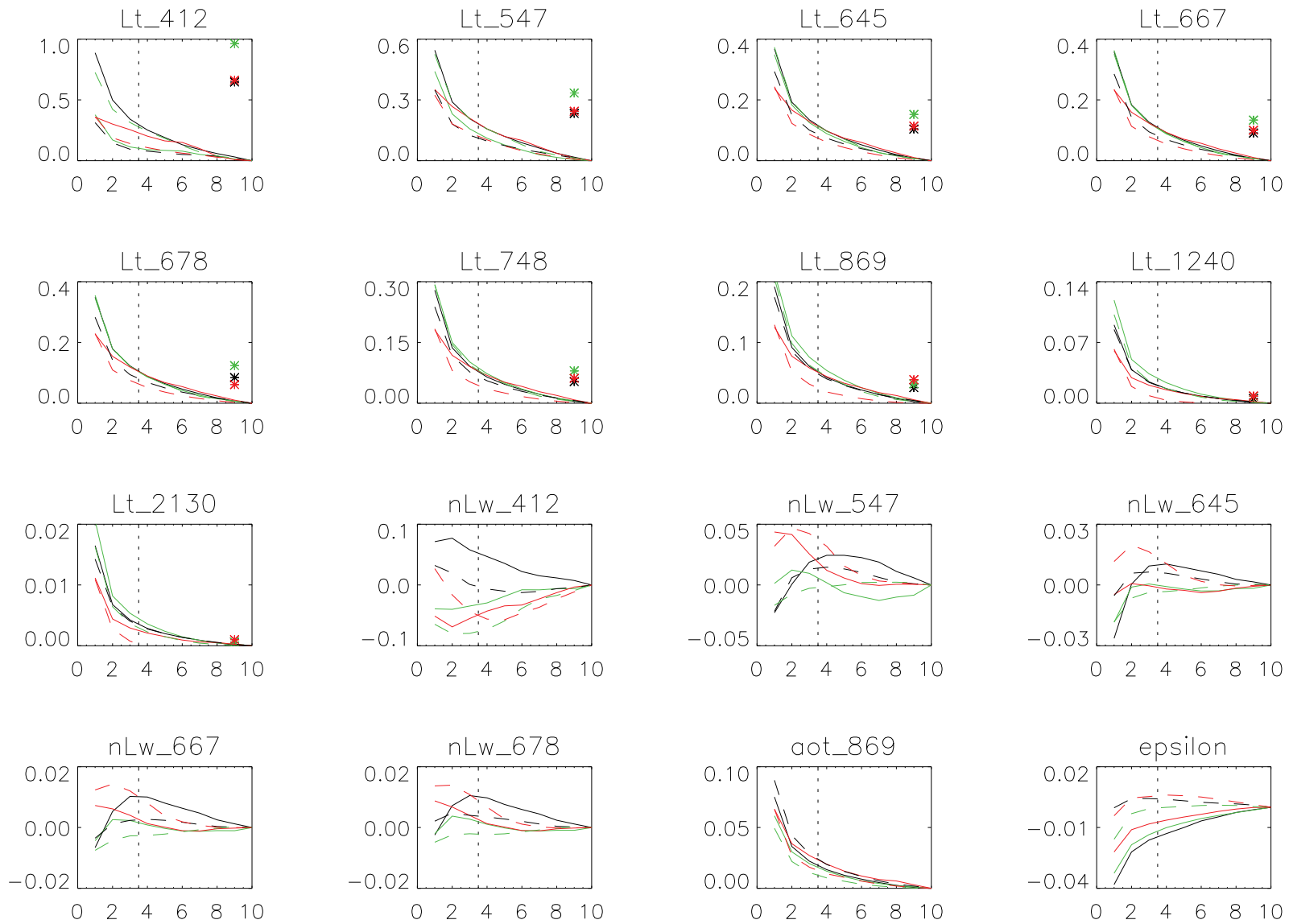

Figure 7. MODIS products as a function of 'Distance to cloud' (approximately in $\mathrm{km}$ ) after subtraction of the product value at a 'Distance to cloud' $=10$. Solid lines are for MODIS Aqua, dashed lines for MODIS Terra. Black, green, and red lines are for granules from table 3, 4, 5, respectively. The stars are plotted at $10 \%$ of the mean product value given in tables 3 to 5 for the Lt products of MODIS Aqua. Vertical dashed line is at 'Distance to cloud'=3.5, all data beyond this will not be masked by the stray light flag. 


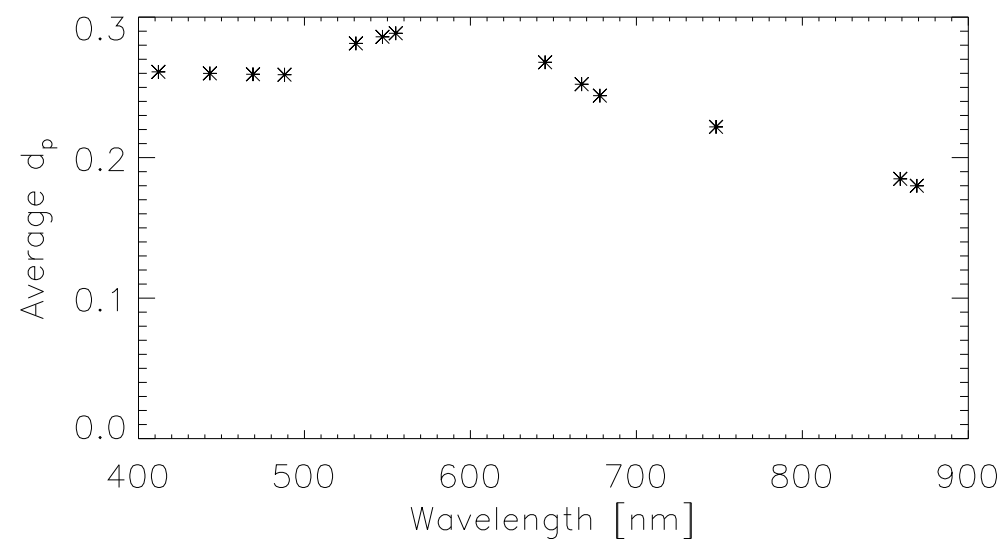

Figure 8. Average degree of polarization $d_{p}$ for an orbit over the Pacific in August 2002 (same as shown in Fig. 9 (d) in Meister et al. ${ }^{9}$ ) as a function of wavelength for MODIS bands with center wavelengths from $412 \mathrm{~nm}$ to $869 \mathrm{~nm}$. The average was calculated for all pixels of this orbit with a valid chlorophyll retrieval.

is highest there, resulting in a higher confidence in the retrievals. An overview of the crosscalibration results is shown for MODIS Terra in another paper of this conference, ${ }^{13}$ the MODIS Aqua results in Meister et al., $2011 .{ }^{14}$ There are several issues worth noting regarding Fig. 9:

- There is good qualitative agreement of the early mission retrievals with the crosscalibration method and the prelaunch measurements.

- The crosscalibration of MODIS Terra to SeaWiFS and MODIS Terra to MODIS Aqua are in good agreement as well, with both showing a slight decrease at the end of the mission, and an average difference of about 0.01 .

- The seasonal oscillations in $m_{13}$ for MODIS Terra and Aqua are opposite in phase. This suggests that there is a problem regarding the geometry of the solar angle with either the polarization model (i.e. the calculation of the Stokes vector for the TOA radiance) or the atmospheric correction.

Fig. 10 shows the same data for the beginning of scan. It can be seen that there are strong correlations between the $m_{12}$ of MODIS Aqua and Terra. Note that for the end of scan (Fig. 9), there is almost no correlation for the $m_{12}$ between sensors. The seasonal oscillations in $m_{13}$ for MODIS Terra and Aqua are again opposite in phase, but the effect is not as clear as in Fig. 9. The seasonal variations seen in the crosscalibration polarization sensitivity coefficients are not yet understood and require further investigation.

In both Figures 9 and 10 the correlation between the results for bands 13 and 14 is very strong for the crosscalibrations to SeaWiFS, for both MODIS Aqua and Terra (compare the black lines in the left column to those in the right columns, and the green lines in the left column to those in the right columns). This suggests that noise in bands 13 and 14 is not a driver for the noise in the $m_{12}$ and $m_{13}$ retrievals of the crosscalibration method.

\section{CONCLUSIONS}

A dual gain design was used in SeaWiFS, with high gains for the ocean radiances and low gains for the cloud radiances. The two gains differed by a factor of approximately 20 (see Fig. 1 in Eplee et al. ${ }^{5}$ ), and provided meaningful benefits. The gain difference between 'high' and 'low' for MODIS bands $13(667 \mathrm{~nm})$ and $14(678 \mathrm{~nm})$ is only about a factor of 2, and the benefit gained is marginal. There is an increase in SNR of up to $25 \%$, but this advantage cannot be fully used because there are other error sources that dominate the ocean color products 
(e.g. calibration and stray light). Therefore, the OBPG decided to use the low gain versions of bands 13 and 14 only, because their larger dynamic range increases coverage. The high gain bands could be used only for low radiances, but the small benefit of the increased SNR (improvement of less than 0.02\%) would be outweighed by the challenge of calibrating the high gain bands relative to the low gain bands (current relative calibration accuracy is about $0.1 \%$ [private communication from MCST]).

To assess the radiometric quality for ocean color applications of bands 13 and 14 relative to the other MODIS bands, the noise in a scene was characterized by assuming that the average of a $5 \times 5$ box of open ocean radiances gives an estimate of the true value for the central pixel of that box. The noise in the water-leaving radiances of ocean bands 13 and 14 of about $7 \%$ is significantly lower than the noise of the spectrally nearest land band (band 1, after averaging to $1 \mathrm{~km}$ resolution) of more than $10 \%$. This difference is larger than the difference of the noise in the water-leaving radiance of ocean band $12(547 \mathrm{~nm}$, about $1.5 \%)$ relative to land band $4(555 \mathrm{~nm}$, about $2.4 \%$ ), and considerably larger than the difference of the noise in the water-leaving radiance of ocean band $9(443 \mathrm{~nm}$, about $0.9 \%)$ relative to land band $3(469 \mathrm{~nm}$, about $1.0 \%)$. Note that the metric used here includes natural variation and very different levels of water-leaving radiances, which account for most of the differences at different wavelengths. Higher wavelengths have lower radiances and therefore higher noise on a relative basis. The increase of noise with wavelength is probably related to a larger natural variation (at higher wavelengths, the spatial distribution of aerosols has a larger impact; at shorter wavelengths, homogeneous Rayleigh scattering reduces noise) and a larger stray light impact from clouds as demonstrated in section 5 (the contrast between bright clouds and dark oceans increases with wavelength).

The same method was used to calculate the noise at the TOA radiance level. For the red bands, the noise (using the above metric) is about $0.2 \%$ to $0.3 \%$, almost twice as high as for the blue bands. The metric used here did not show less noise for the high gain bands relative to the low gain bands. The noise due to natural variation and instrument noise combined (using the above metric) is about twice as large as instrument noise alone (using noise characterization from solar diffuser measurements) at 412nm, 5 to 6 times as large at $667 \mathrm{~nm}$ and $678 \mathrm{~nm}$, and 8-12 times as large at $869 \mathrm{~nm}$. The same caveats as in the paragraph above apply here.

At $1240 \mathrm{~nm}$ and $2130 \mathrm{~nm}$, the noise is on the order of several percent. The ocean radiances are approximately a tenth of the typical radiances for the land applications for these two bands, and their SNR is insufficient at these low light levels.

For the 2006 scene, SeaWiFS MLAC data is available and was included in the analysis. Generally, the TOA radiance noise is similar to MODIS, but the ocean color products of SeaWiFS are significantly noisier, even compared to the MODIS land bands at $1 \mathrm{~km}$ resolution. Note that at their native resolution $(250 \mathrm{~m}$ or $500 \mathrm{~m})$, the MODIS land band noise is expected to be higher than at the $1 \mathrm{~km}$ resolution analyzed here.

The noise was evaluated for only three scenes in this paper, which is not sufficient to produce representative averages, as can be seen in the large spread of values between tables 3,4 , and table 5, especially for the higher wavelengths. The large spread suggests that a more sophisticated approach may need to be developed; apparently, the large number of samples ( $\geq 17,0005 \times 5$ boxes per scene) was not sufficient to eliminate scene specific noise characteristics.

The polarization sensitivity of the bands 13 and 14 is generally low, and the values retrieved from the crosscalibration to SeaWiFS agree reasonably well with the prelaunch measurements. This leads to a relatively high confidence in our knowledge of the MODIS polarization correction for those two bands. The polarization correction of bands 13 and 14 is important for ocean color applications, because the average degree of TOA radiance polarization of bands 13 and 14 is about $25 \%$, similar to that in the blue wavelengths.

\section{ACKNOWLEDGMENTS}

We want to thank our colleagues from OBPG for their support, especially Sean Bailey, and from the MCST, especially Junqiang Sun and Jack Xiong. This work was funded by the NASA MODIS Science Team. 



Figure 9. The straight black line shows the prelaunch $m_{12}$ (top) and $m_{13}$ (bottom) for MODIS Terra, the straight green line for MODIS Aqua. The zagged black line shows the $m_{12}$ and $m_{13}$ retrieved from the crosscalibration of MODIS Terra to SeaWiFS, the zagged green line for MODIS Aqua to SeaWiFS. The red diamonds show the $m_{12}$ and $m_{13}$ retrieved from the crosscalibration of MODIS Terra to MODIS Aqua. Left column is for band 13, right column for band 14 . All values are for mirror side 1 and detector 1 at a scan angle of $+45^{\circ}$ (frame 1231).
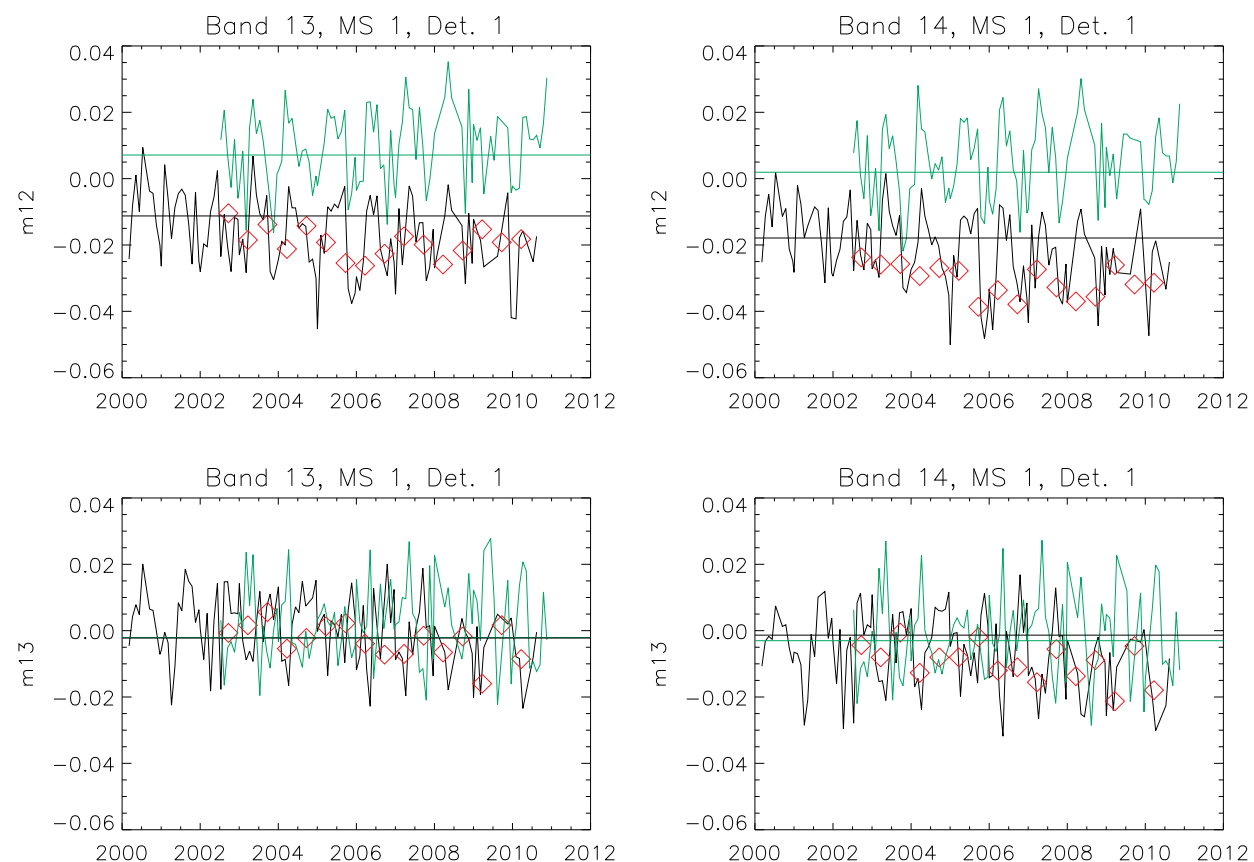

Figure 10. Same as Fig. 9, but at a scan angle of $-45^{\circ}$ (frame 122). 


\section{REFERENCES}

[1] Barnes, W. L., Pagano, T. S., and Salomonson, V. V., "Prelaunch characteristics of the moderate resolution imaging spectroradiometer (MODIS) on EOS-AM1," IEEE Transactions on Geoscience and Remote Sensing 36(4), 1088-1100 (1998).

[2] Franz, B. A., Werdell, P. J., Meister, G., Bailey, S. W., Eplee, R. E., Feldman, G. C., Kwiatkowska, E., McClain, C. R., Patt, F. S., and Thomas, D., "The continuity of ocean color measurements from SeaWiFS to MODIS," Proc. SPIE 5882(1), 58820W, SPIE (2005).

[3] Esaias, W. E., Abbot, M. R., Barton, I. J., Brown, O. B., Campbell, J. W., Carder, K. L., Clark, D. K., Evans, R. H., Hoge, F. E., Gordon, H. R., Balch, W. M., Letelier, R., and Minnett, P. J., "An overview of MODIS capabilities for ocean science observations," IEEE Transactions on Geoscience and Remote Sensing 36, 1250-1265 (1998).

[4] Behrenfeld, M. J., Westenberry, T. K., Boss, E. S., O’Malley, R. T., Siegel, D. A., Wiggert, J. D., Franz, B. A., McClain, C. R., Feldman, G. C., Doney, S. C., Moore, J. K., Dall'Omo, G., Milligan, A. J., Lima, I., and Mahowald, N., "Satellite-detected fluorescence reveals global physiology of ocean phytoplankton," Biogeosciences 6, 779-794 (2009).

[5] Eplee, R. E., Patt, F. S., Franz, B. A., Bailey, S. W., Meister, G., and McClain, C. R., "SeaWiFS on-orbit gain and detector calibrations: effect on ocean products," Applied Optics 46(27), 6733-6750 (2007).

[6] Nishihama, M., [MODIS Level 1A Earth Location: Algorithm Theoretical Basis Document Version 3.0], NASA, Goddard Space Flight Center (1997).

[7] Esposito, J. A., Xiong, X., Wu, A., Sun, J., and Barnes, W. L., "Modis reflective solar bands uncertainty analysis," Earth Observing Systems IX 5542(1), 448-458, SPIE (2004).

[8] Meister, G. and McClain, C. R., "Point-spread function of the ocean color bands of the Moderate Resolution Imaging Spectroradiometer on Aqua," Applied Optics 49(32), 6276-6285 (2010).

[9] Meister, G., Kwiatkowska, E. J., Franz, B. A., Patt, F. S., Feldman, G. C., and McClain, C. R., "ModerateResolution Imaging Spectroradiometer ocean color polarization correction," Applied Optics 44(26), 5524$5535(2005)$.

[10] Meister, G., Kwiatkowska, E. J., and McClain, C. R., "Analysis of image striping due to polarization correction artifacts in remotely sensed ocean scenes," Proc. SPIE 6296(1), 629609 (2006).

[11] Xiong, X., Sun, J., Xie, X., Barnes, W. L., and Salomonson, V. V., "On-orbit calibration and performance of Aqua MODIS reflective solar bands," IEEE Transactions on Geoscience and Remote Sensing 48(1), 535-546 (2010).

[12] Kwiatkowska, E. J., Franz, B. A., Meister, G., McClain, C. R., and Xiong, X., "Cross calibration of ocean-color bands from Moderate-Resolution Imaging Spectroradiometer on Terra platform," Applied Optics 47(36), 6796-6810 (2008).

[13] Meister, G. and Franz, B. A., "Adjustments to the MODIS terra radiometric calibration and polarization sensitivity in the 2010 reprocessing," in [Earth Observing Systems XVI], Butler, J. J. and Xiong, J., eds. (2011).

[14] Meister, G., Franz, B. A., Kwiatkowska, E. J., and McClain, C. R., "Corrections to the calibration of MODIS Aqua ocean color bands derived from SeaWiFS data," IEEE Transactions on Geoscience and Remote Sensing, accepted (2011). 